\title{
Ateliers A1 à A4
}

\section{Atelier A1 - Déficience intellectuelle et maladies rares : quelles perspectives pour la prise en charge et les traitements?}

\section{Pierre Sarda, CHRU de Montpellier}

Cet atelier a été marqué par deux particularités : le faible nombre de personnes présentes (une quinzaine) et la très grande intensité des discussions. J'espère qu'il sera reconduit dans les années à venir.

Cet atelier a permis d'aborder les nouvelles perspectives de prise en charge des enfants présentant une déficience intellectuelle. Pendant des années, la personne avec une déficience intellectuelle a simplement été prise en charge de manière peu spécifique par les kinésithérapeutes et les orthophonistes essentiellement; à l'âge adulte, elle intégrait une structure d'adulte le plus souvent sans poursuite d'une «stimulation » adaptée. Grâce aux connaissances neurophysiologiques et à la neurobiologie, des prises en charge très spécifiques sont en train d'émerger. Dans la majorité des cas, une déficience intellectuelle est due à un gène déficient. Celui-ci ne rabaisse pas l'ensemble des capacités cérébrales à des valeurs inférieures à la normale qui serait mesuré par un $\mathrm{Ql}$ abaissé de manière uniforme ! Un gène déficient a des actions dans certaines fonctions du cerveau. Certaines sont altérées, responsables de la déficience observée, tandis que d'autres sont conservées, voire même améliorées par rapport à une personne non déficiente.

La vision que nous devons avoir en 2015 des personnes présentant une déficience intellectuelle est qu'elles ont certes des déficiences à rééduquer mais surtout des capacités à valoriser! Chaque maladie étant différente, chaque enfant doit bénéficier d'une prise en charge adaptée à sa pathologie génétique.

L'atelier a permis à quatre intervenants de présenter des techniques qui viennent d'être découvertes, mais qui auront une importance majeure à l'avenir.

Une technique simple, aisément généralisable, consiste à utiliser une tablette tactile. Des centaines d'applications - jeux, tracé, écriture, mémoire visuelle immé- diate, etc. - sont utilisées pour rééduquer des fonctions défaillantes (motricité fine, langage, mémoire, calcul...), mais il est très difficile d'avoir une vision des applications existantes. La présentation a été faite par Madame Bilgi Ancel de l'IME de La Salette à Bédarieux qui a décidé d'utiliser des tablettes tactiles sur une vingtaine d'enfants. Ces appareils proposent trois niveaux de programmes. Ceux-ci sont utilisés pendant plusieurs mois. À la fin de l'utilisation d'une strate du programme, l'enfant est évalué. Dans la majorité des cas, ces tablettes sont proposées par les orthophonistes. Comme elles sont mobiles, elles peuvent être utilisées par un professionnel, la famille ou l'enfant. Cet outil aura un grand avenir. Une réflexion sur l'évaluation des résultats obtenus devrait être initiée. En outre, un site web devrait être créé pour faciliter la circulation dans cette « jungle » des logiciels. Enfin, il serait intéressant que des entreprises conçoivent des programmes adaptés à des déficits très spécifiques d'enfants déficients.

Caroline Demily étant absente, nous avons pu cependant présenter ses données. Cette psychiatre de Lyon travaille actuellement sur les remédiations cognitives. Des programmes informatiques sont créés dans des laboratoires de neurosciences pour améliorer une fonction très particulière, comme la reconnaissance des émotions faciales des personnes que l'on a en face de soi. Caroline a créé des programmes informatiques qui sont utilisés 45 minutes par semaine pendant quatre mois. Des personnes atteintes de délétion 22q11 (Association Génération 22) ont pu, après ces remédiations, reprendre une vie sociale plus adaptée (se promener en ville, par exemple).

L'évaluation réelle de ces programmes sophistiqués devrait être formalisée. En outre, l'utilisation de ces outils pourrait être généralisée et mise en place non seulement par les familles ou les professionnels paramédicaux de proximité, mais aussi dans des structures médicoéducatives des jeunes (IME, IMPro...) ou des centres de vie des adultes (ESAT, foyers de vie, ateliers occupationnels, MAS, FAM...).

Enfin, Monsieur Xavier Liogier Dardhuy, responsable des projets scientifiques, travaillant dans le laboratoire pharmaceutique Roche, a présenté un projet en cours de développement dans la trisomie 21. La molécule élaborée par le laboratoire a pour but de diminuer le déficit intellectuel des enfants trisomiques. Monsieur Xavier Liogier Dardhuy nous a expliqué comment Roche procède, à partir des connaissances neurobiologiques récentes apportées par les neurosciences, pour élaborer une molécule possiblement thérapeutique pour une maladie génétique très précise. Les résultats de cet essai thérapeutique, chez les personnes trisomiques âgées de 12 à 30 ans, sont en attente pour l'année 2016.

De son côté, Mme Poher, membre de l'Union nationale des associations de parents, de personnes handicapées mentales et de leurs amis (UNAPEI), a fait écho aux différents outils présentés. Elle a insisté sur 
la capacité des jeunes à s'approprier l'outil numérique. Elle propose ainsi d'encourager ces techniques, de les évaluer et de mieux les intégrer dans des programmes pédagogiques notamment. Concernant les remédiations cognitives, Mme Poher a insisté sur l'importance et le bénéfice d'un bilan neuropsychologique, car, trop rare encore, il permet de faire le point sur ce que la personne est capable d'apprendre, sur ses fonctions préservées et sur les fonctions qu'il faudra rééduquer. Enfin, Mme Poher a souligné la nécessité de rechercher la pleine adhésion des personnes et de leurs proches dans le cadre d'essais thérapeutiques, avant, pendant, et après leur mise en œuvre. $\varepsilon$ t tout particulièrement après, lorsque l'essai n'est pas positif, il importe de prendre le temps de partager les résultats dans un climat de confiance réciproque. II s'agit, pour Mme Poher, d'élaborer et de savoir mettre en œuvre aujourd'hui des réponses aux constats et aux manques, connus et partagés, dans un souci d'égalité d'accès de tous au progrès, dans le respect d'une médecine fondée sur les preuves et de règles éthiques strictes.

\section{Atelier A2 - Mise en place des filières maladies rares: quelles sont les clés de la réussite?}

\section{Laure Druetta, Genzyme}

Une cinquantaine de personnes, dont dix représentants d'associations de patients, dix représentants de la partie académique et clinique, huit représentants industriels et plus de vingt représentants de la coordination des filières ont participé à cet atelier.

\section{Claude Desnuelle, CHU de Nice}

La question posée dans le cadre de cet atelier était ambitieuse. Le dispositif mis en place par la circulaire du 23 juillet 2013 a permis de définir des objectifs généraux sur l'amélioration de la qualité de vie des personnes atteintes de maladies rares, ainsi que sur le décloisonnement, car ces filières doivent être un lieu de regroupement, d'identification et d'échanges entre un ensemble d'acteurs intervenant autour de la prise en charge de personnes atteintes de maladies rares. Pour atteindre ces objectifs généraux, des objectifs opérationnels ont été identifiés. Ils visent notamment à renforcer l'expertise - en particulier la pluridisciplinarité, qui constitue un mot-clé dans les centres de référence -, à formuler des recommandations de bonnes pratiques sous forme de PNDS ou de recommandations de fonctionnement, à aller dans le sens de la recherche et de la coordination des projets de recherche, à prendre une large part dans l'épidémiologie - c'est-àdire le développement de bases de données -, à assurer des formations, et à orienter les regroupements vers l'Europe, en particulier vers les réseaux européens de référence.

Les facteurs clés de succès suivants permettent d'atteindre ces objectifs :

\section{- La volonté politique}

Le pilotage des filières est assuré par le ministère de la Santé, alors que les objectifs s'inscrivent dans plusieurs domaines (santé, recherche, partages internationaux). L'association d'autres ministères serait peut-être nécessaire pour ne pas limiter ces filières à la vision santé et l'organisation de la santé. La volonté politique vis-à-vis des personnes atteintes de maladies rares a posé question, car les participants s'interrogent beaucoup sur la mise en œuvre d'un ze PNMR. Si celui-ci ne voit pas le jour, les objectifs des filières ne seront sans doute pas concrétisés jusqu'au bout.

\section{- La communication}

La communication s'opère entre nos tutelles réglementaires et les acteurs. Des stratégies, des perspectives et des plans d'action doivent être définis. Un premier appel à plan d'action a été lancé en juin 2015. II a montré que la communication devait être précisée et améliorée, à travers notamment davantage de transversalité, de décloisonnement et de lisibilité. Un véritable continuum de communication doit exister entre les tutelles de santé et tout ce qui compose ces filières (le sanitaire, les laboratoires de diagnostic, le médico-social, les associations, les sociétés savantes, la recherche fondamentale, les industries - qui ne sont pas mentionnées dans la circulaire de 2013).

\section{- L'harmonisation}

II nous est demandé dans les objectifs de travailler sur l'épidémiologie, sur des bases de données. Hier, nous avons vu qu'un nombre impressionnant de bases de données existaient dans la prise en charge des maladies rares et dans les différents centres. II est certain que ces bases doivent être harmonisées. La notion de codage est essentielle. Les acteurs des filières ont de fortes attentes et inquiétudes vis-à-vis de cette harmonisation. Par ailleurs, les acteurs présents ont estimé que les réseaux européens de référence souffraient d'un déficit de communication. Le manque de présence de la France au niveau européen suscite une réelle inquiétude. Enfin, les associations doivent jouer un rôle majeur en matière d'harmonisation. Une représentante d'une association a expliqué qu'elle ne se reconnaissait pas dans les filières existantes. L'harmonisation concerne donc l'organisationnel, mais aussi l'associatif.

\section{- La gestion et le budget}

Une inquiétude a été exprimée concernant la gestion et les budgets. Les CHU se sont vus confier des missions nationales de gestion de budget qu'ils sont incapables de gérer. Pour résoudre ce problème, des référents filières régionaux devraient sans doute être désignés au niveau des ARS.

\section{- L’identification}

L'identification est très importante. La visibilité et les missions des centres de référence et des filières ont été rappelées. Les rôles de chacun des acteurs doivent être respectés dans une transparence et une confiance transversale.

\section{De la salle}

Des solutions ont-elles été discutées durant l'atelier? 
Claude Desnuelle

Les solutions consistent à améliorer les points listés. Ceux-ci sont actuellement identifiés par les acteurs comme des difficultés rencontrées dans le fonctionnement.

\section{Christian Deleuze}

Hier, a été abordée la question de la non-harmonisation du dispositif des Réseaux Européens d'Excellence avec les principes du dispositif français maladies rares. En avez-vous discuté au cours de l'atelier?

\section{Claude Desnuelle}

Ce sujet a été abordé dans le cadre de l'atelier, mais il est plus de la responsabilité des animateurs de filière ou des responsables de centres de référence. Après échanges, il est apparu que les hôpitaux eux-mêmes devaient se faire intégrer dans ces réseaux. Un travail d'information vis-à-vis des centres de référence et des acteurs doit être mené pour qu'ils se regroupent et intègrent ces filières. Dans ce dispositif, les États valident les demandes, c'est donc aux Autorités de santé de chaque État à qui il revient de faire des choix.

\section{De la salle}

Des initiatives ont-elles été formulées lors de l'atelier?

\section{Claude Desnuelle}

Pour l'heure, nous ne disposons pas de remèdes. Le bilan d'un an de fonctionnement fait apparaître quelques défauts. Nous devons être vigilants vis-à-vis des inquiétudes exprimées. Aucune initiative de correction de défauts n'a été formulée au cours de l'atelier.

\section{Atelier A3 - Errance diagnostique : comment informer et accompagner le malade?}

\section{Anne-Sophie Lapointe, Vaincre les Maladies Lysosomales, filière AnDDI-Rares \\ Cet atelier présentait une grande complémentarité entre Maladies Rares Info Services, la filière AnDDI-Rares et le laboratoire Shire. L'errance diagnostique est un réel enjeu pour tous les acteurs dans le champ des maladies rares. \\ Le Larousse définit ainsi la notion d'errance : "action d'aller sans direction précise, de chercher son chemin ». Au regard du diagnostic, l'errance correspond à un délai significatif entre les premiers symp- tômes et le diagnostic. Il existe également une problématique dans les enjeux d'absence de diagnostic versus l'errance. Deux types d'errance ont été mis en évidence au cours de l'atelier : l'errance liée au fait que le diagnostic n'est pas posé alors que la maladie est connue; l'errance liée au fait que la maladie n'est pas encore connue. \\ Les enjeux varient selon les points de vue de chacun:}

\section{- Le point de vue de la personne malade}

Nous avons eu beaucoup de retours de la salle sur l'errance diagnostique par rapport à la personne malade, avec la nécessité de l'accompagnement psychologique par le centre de référence et la famille. La psychologue Éva Toussaint de Bordeaux a souligné
I'importance, en particulier en cas d'errance diagnostique, de la continuité de l'accompagnement de la personne par le centre de référence de génétique.

\section{- Le point de vue médical}

Beaucoup sont intervenus pour expliquer que le médecin généraliste devrait pouvoir poser le diagnostic. Ce professionnel de santé ne pouvant connaître les 7000 maladies rares, il serait préférable de travailler sur la culture du doute. Certains ont souligné la mauvaise connaissance des ressources maladies rares par les professionnels hors maladies rares. Un travail de communication/formation sur les maladies rares devrait donc être mené en direction des médecins généralistes et spécialistes, en particulier en ambulatoire. À cette fin, le projet de mettre en place une stratégie pluriannuelle et coordonnée de toutes les ressources a ainsi été évoqué.

\section{- Le point de vue sociétal}

Les conséquences de l'errance diagnostique ont également été abordées au cours de l'atelier. Le lien de confiance avec la personne malade me semble important. Trop souvent, des personnes arrivent dans nos associations après une longue errance diagnostique, et sont un peu en révolte vis-à-vis du système. Une longue errance constitue un accroc dans la relation de confiance, y compris dans le cadre des essais cliniques.

\section{- Le point de vue des chiffres}

L'expérience (enquête de l'Observatoire des Maladies Rares) montre que plus de 4 fois sur 10, le diagnostic est réalisé dans l'année. Plus de 3 fois sur 10 , le diagnostic est posé entre 1 à 5 ans. Plus de 2 fois sur 10 , l'errance est supérieure à 5 ans.

La formation constitue un levier essentiel pour développer la culture du doute. II importe que les professionnels se posent les bonnes questions et sachent orienter vers les personnes ad hoc. Les outils (plaquettes informatives, blogs, signes d'appel) constituent un autre type de levier.

Les nouvelles techniques de séquençage ont été abordées au cours de l'atelier et présentées par un film: http://www.anddi-rares.org/axes/informer/supportsnumeriques-de-la-filiere-anddi-rares.html. Ces nouvelles techniques devraient être utilisées pour les personnes concernées par une très longue errance diagnostique. Des verrous liés au coût de ces techniques sont en train de sauter. Au cours de la décennie à venir, le prix du séquençage sera très accessible. Cette technique doit toutefois faire l'objet d'un accompagnement spécifique.

De nombreuses questions ont été posées au cours de l'atelier. La notion d'errance ne se limite pas au diagnostic ; elle concerne également le parcours de vie du 
malade et l'articulation médicosociale. Un accompagnement multidisciplinaire est indispensable. Dans ce domaine, les centres de référence jouent un rôle essentiel, car l'impact humain est grand.

La question de la caractérisation génotype-phénotype a été posée, car les maladies rares présentent une grande hétérogénéité ; en outre, les résultats cliniques peuvent être très différents pour une même anomalie. Le travail de mise en commun de l'information entre les professionnels de santé a été mis en avant.

Un travail est en train d'émerger entre Maladies Rares Info Services et la filière AnDDI-Rares. Les personnes sans-diagnostic représentent la moitié de la file active de la filière. Comme aucune association ne les représente, la question de leur accompagnement se pose. Maladies Rares Info Services recevant chaque année plus de 1100 demandes de personnes qui n'ont pas de diagnostic, il a été décidé avec la filière AnDDI-Rares de créer une offre de services dédiée pour apporter une aide concrète aux patients et à leurs proches.

L'errance pose un questionnement éthique. Réduire le temps d'errance constitue en effet un acte de bienveillance par rapport aux personnes. L'absence de nom de maladie fait obstacle à la recherche et au développement d'un traitement thérapeutique - ce qui pose un problème d'équité et d'accès au traitement. En l'absence de diagnostic, la famille ne peut pas faire de choix. Enfin, il faut veiller à la bonne interaction entre le soin et la recherche.

\section{Antoine Bernasconi, laboratoire Orphan Europe}

Je suis surpris que la question du dépistage néonatal n'ait pas été abordée au cours de cet atelier. II s'agit en effet d'un des outils permettant de réduire l'errance diagnostique. En France, seules cinq maladies sont dépistées à la naissance. Dans d'autres pays européens, un dépistage néonatal beaucoup plus large a été mis en place. Pourquoi la France n'utilise-t-elle pas pleinement cet outil, notamment pour dépister les maladies progressives?

\section{Anne-Sophie Lapointe}

Au cours de l'atelier, nous avons abordé la question de l'amélioration des outils de diagnostic. Pour la maladie de Pompe, le diagnostic était initialement réalisé avec des biopsies musculaires; il est maintenant réalisé avec le test de Guthrie. Le diagnostic est maintenant posé par des techniques moins invasives et plus faciles à mettre en place rapidement.

Le dépistage néonatal pose la question du lien entre génotype et phénotype. En outre, il interroge sur l'explication des résultats du dépistage à donner aux parents alors que la maladie ne se déclarera pas forcément ou alors très tard à l'âge adulte avec une forme modérée. Quand le lien entre génotype et phénotype sera clairement caractérisé, la solution du dépistage néonatal pourra être déployée à une plus grande échelle.

\section{Christian Deleuze}

Je me demande pourquoi les pays européens ont une approche si différente dans ce domaine. L'Italie a fait le choix de l'action, alors que la France a opté pour l'inaction. Nous ne savons pas encore lequel de ces deux pays a fait le bon choix, mais il serait intéressant de savoir si la France fait les efforts nécessaires pour apprendre de l'expérience italienne.

\section{Pierre-Henri Gandon, Coopération Santé}

Des disparités de prise en charge de diagnostic existent. Sur le plan territorial, des départements ou des régions sont-ils sinistrés? Par ailleurs, je m'occupe de l'Association MG France, qui est très favorable à la création d'un cycle de formation et d'information. L'administrateur qui représente Coopération Santé a vu quatre cas en 40 ans de carrière professionnelle. II ne savait rien sur ces maladies.

\section{Anne-Sophie Lapointe}

Les différences sont peut-être moins entre les territoires qu'entre les structures hospitalières avec spécialistes et les structures avec médecins généralistes. La solution de MG France consiste à passer par un syndicat de médecins généralistes et à former l'ensemble des généralistes. Les médecins doivent être capables d'orienter la personne malade vers le bon centre.

\section{Ségolène Aymé}

Je m'étonne que la solution d'aide au diagnostic informatisé n'ait pas été évoquée dans la première partie de l'atelier. C'est pourtant «la » solution. Dans le domaine de la génétique, nous utilisons cet outil depuis 30 ans. Compte tenu de la multiplicité des syndromes existants, nous ne saurions travailler sans cet outil. Celui-ci ne présente pas encore une convivialité adaptée pour être utilisé par les médecins en ville. L'offre d'aide au diagnostic doit être développée, car elle constitue la voix d'avenir. Le système d'aide au diagnostic disponible sur Orphanet est l'une des fonctionnalités du site les plus utilisées.

\section{Anne-Sophie Lapointe}

Cette solution n'a effectivement pas été abordée dans l'atelier.

\section{Pierre Sarda}

Une personne sur 20 a une maladie rare. En 40 ans de carrière, un médecin ne voit pas seulement quatre cas; le problème est que les autres cas ne sont pas repérés. Les outils utilisés par les généticiens tous les jours devraient être démocratisés pour les médecins.

\section{Christian Deleuze}

Dans un département du centre de la France, $15 \%$ des médecins généralistes ont connaissance des PNMR. Les outils existent, mais ils ne sont pas suffisamment 
connus des médecins généralistes. Un travail d'information/formation reste à faire.

\section{De la salle}

Une articulation avec le cluster sur les services à la personne sociale et médico-sociale permettrait d'expérimenter des détections et des prises en charge innovantes.

\section{Anne-Sophie Lapointe}

La sensibilisation des acteurs médico-sociaux (IME, MAS, etc.) à la problématique des maladies rares est très importante. II faut savoir que des personnes atteintes de la maladie de Niemann-Pick type C sont hospitalisées en hôpitaux psychiatriques, alors qu'un traitement améliorerait considérablement leur prise en charge. Cet exemple souligne l'importance pour les médecins et les psychiatres de ne pas limiter leur diagnostic aux malades psychiques mais ils doivent être capables de se poser la question : et si c'était une maladie génétique?

\section{Atelier A4 - Le mécanisme de l'évaluation et de la fixation des prix des médicaments orphelins est-il adapté ?}

\section{Neil Bernard, Genzyme}

Nous n'avons pas eu la chance de bénéficier du point de vue des autorités d'évaluation lors de cet atelier. Avec Christophe Duguet, Antoine Bernasconi et les participants dans la salle, nous avons évoqué l'ensemble des problématiques pouvant se poser dans les méthodologies d'évaluation de fixation du prix et de financement des médicaments orphelins et plus globalement des médicaments destinés à traiter des maladies rares. Nous avons également réfléchi aux freins actuels à la mise à disposition de ces traitements. L'atelier a réuni un assez grand nombre de participants. Les échanges ont été vifs, ce qui prouve leur intérêt pour ces sujets. Nous sommes partis d'un constat général. La première attente et le premier défi dans les maladies rares, commun à l'ensemble des acteurs, est la mise à disposition de traitements pour les malades.

Une première remarque : la méthodologie aujourd'hui appliquée est commune à l'ensemble des médicaments; il n'existe pas de mécanisme d'évaluation français spécifique aux médicaments orphelins. La deuxième remarque, plutôt positive : notre système permet, de manière générale, la mise à disposition, le remboursement de la quasi-totalité des médicaments évalués et la prise en charge intégrale du coût de ces médicaments par la société. Des biais existent cependant dans la méthodologie d'évaluation, de fixation du prix et, de manière plus générale, dans la compréhension des maladies rares, ne permettant pas que cette prise en charge soit optimale.

Notre postulat n'était donc pas de savoir si notre système est idéal (nul système ne l'est) ou s'il est perfectible, mais plutôt d'envisager les solutions permettant d'optimiser les conditions d'évaluation, de mise à disposition et de prise en charge de ces traitements.

La première difficulté inhérente à ces médicaments réside dans les biais techniques et méthodologiques dans leur évaluation. Les biais techniques sont liés à la rareté des patients, à la méconnaissance de l'histoire naturelle de la maladie (avant l'arrivée d'un traitement) et à la démonstration de preuve. Le vrai enjeu est donc de définir les critères méthodologiques permettant, d'une part, de démontrer l'efficacité de médicaments - notamment en termes de ralentissement de l'évolution de ces maladies progressives - et, d'autre part, de permettre l'accès des malades à ces traitements.

À titre d'exemple, un test de marche de six minutes démontrant une progression de 25 mètres du malade peut-il être considéré par les autorités comme un critère suffisant pour avoir un niveau de preuve et une notation importants permettant l'accès à ce traitement à un niveau d'évaluation suffisant ? La distance de 25 mètres ne représente rien en termes méthodologiques, mais elle peut faire une différence considérable dans le quotidien de certains patients. La notion de qualité de vie est difficilement démontrable, mais elle est essentielle pour ces personnes.

La question des interactions entre l'évaluation européenne et l'évaluation française a été posée lors de l'atelier. Les méthodologies d'évaluation jusqu'à I'AMM sont définies de manière internalisée, notamment avec les autorités européennes, sur des critères faisant l'objet d'un accord. Toutefois, les laboratoires ont parfois l'impression que le dossier d'AMM est intégralement réévalué par l'autorité française. Cette pratique est chronophage et pose des interrogations sur l'interaction entre les agences française et européenne.

Les critères d'évaluation aujourd'hui essentiellement basés sur l'efficacité et la tolérance ne permettent pas de reconnaître l'innovation de certains traitements. Si le traitement d'une maladie chronique par voie intraveineuse - à raison d'une perfusion tous les 15 jours tout au long de la vie - pouvait être remplacé par une forme orale, le patient pourrait retrouver une vie quotidienne normale. Or, le traitement oral reçoit une ASMR 5. L'amélioration de la qualité de vie et de la mise au traitement de certains patients est aujourd'hui malheureusement très peu reconnue.

Enfin, il convient de souligner le manque de transparence et de lisibilité des prix. Dans le secteur public, le médicament orphelin est perçu comme cher. Le prix doit-il être fixé en rapportant l'investissement au nombre de patients? Le gain représenté par le traitement doit-il être pris en compte ? Un élément à prendre en compte lorsque l'on évoque le coût de ces traitements est que le prix officiel, publié au Journal Officiel, n'est pas le prix « réel » générant le chiffre d'affaires du laboratoire. Un article de l'accord cadre définissant les relations entre le LEદM (représentant les entreprises du médicament) et le CEPS (Comité 
économique fixant les prix des médicaments) définit la notion de plafonnement de chiffre d'affaires dans les maladies rares. Ce mécanisme, évoqué hier, précise que si le laboratoire dépasse un plafond de chiffre d'affaires fixé avec le CEPS (à savoir un prix $X$, multiplié par un nombre de patients $Y$, préétablis), il doit intégralement reverser l'excédent à l'assurance-maladie. Le prix officiel n'est donc pas le prix réellement payé par les pouvoirs publics.

Les conclusions et propositions suivantes ont été formulées au cours de l'atelier:

- ne pas remettre en cause les évaluations européennes, en particulier sur des questions méthodologiques;

- démocratiser davantage le système d'évaluation, en le rendant plus collaboratif, plus prédictible et plus encourageant ;

- faciliter l'évaluation du bénéfice thérapeutique d'un traitement à partir de toutes les sources d'information disponibles;

- intégrer d'autres critères d'évaluation, afin d'encourager toutes les formes d'innovations utiles aux malades;

- accepter une «prise de risque » pour les maladies rares (compte tenu du manque de données et de recul lors de l'arrivée du traitement, le LદદM encourage une évaluation et une fixation de prix conditionnées à une démonstration en vie réelle du médicament).

\section{Laure Jamot}

S'agissant de l'ASMR des molécules développées pour les maladies rares, nous sommes confrontés à un écueil spécifique d'évaluation des coûts directs et indirects de la maladie. La procédure classique de calcul du coût direct consisterait à réaliser une requête auprès du Système national d'information inter-régimes de l'assurance-maladie (SNIIRAM) ou des CRAM. Or, toute requête est refusée en l'absence de traitement spécifique, ce qui impliquerait donc de faire une recherche nominative des soins dont bénéficient des patients identifiés. Mais le Conseil d'État refuse tout « requêtage » nominatif. Dans ce contexte, le coût direct de la maladie ne peut jamais être calculé.

En outre, le coût indirect n'intéresse aucune agence réglementaire. Nous ne sommes pas parvenus à trouver des systèmes cohérents permettant de calculer le coût de prise en charge d'un malade par une famille. Ce coût devrait inclure l'absentéisme au travail, la kinésithérapie, la mise aux normes de la maison et du véhicule, etc. Face au grand flou actuel, aucune solution ne s'offre à nous.

\section{Christian Deleuze}

Vous venez de résumer les thèmes abordés au cours de la première session de ce matin. Pour démontrer la valeur apportée par un traitement, il faudrait comparer les résultats qui seront produits à l'existant. Or, malgré la bonne volonté de tous les acteurs, les données sur la situation antérieure ne sont pas disponibles et les données sur la situation actuelle ne sont disponibles que dans 115 registres parcellaires. La mise en œuvre de modèles économiques est aisée si les données existent. Or, celles-ci font cruellement défaut. Les traitements pour les maladies rares étant coûteux, la démonstration de leur valeur doit être établie dans le temps et dans un modèle collaboratif. Par conséquent, tous les acteurs devraient collaborer pour générer ces données. En général, le financement de la collecte de données repose sur le seul industriel. Celui-ci se voit en outre demander de ne pas trop les regarder et de pouvoir répondre aux questions que posent les progrès apportés par son traitement.

\section{Neil Bernard}

Le consentement collectif à payer suppose un consentement des autorités à mener les démarches réglementaires et administratives nécessaires à l'utilisation d'outils d'évaluation adaptés à ces problématiques.

\section{De la salle}

Va-t-on vers un prix maximum acceptable pour la société pour un médicament orphelin?

\section{Christian Deleuze}

La loi fixe à 50000 euros par patient et par an le prix maximal pour lequel il n'y aurait pas de discussion particulière. Au-delà de ce prix, le CEPS peut décider de fixer une enveloppe maximale qui rémunérera le laboratoire, à condition qu'il s'engage parallèlement à traiter tous les patients qui devraient bénéficier de ce traitement. Comme la population des malades n'est pas précisément connue au moment du lancement d'un médicament, le laboratoire doit accepter de se lancer dans un véritable pari.

Si nous admettons que les autorités de santé sont une émanation du pouvoir politique, lequel représente la population, il nous appartient de décider collectivement. II faut éviter que ces décisions soient prises dans des cabinets obscurs.

\section{Neil Bernard}

Pour compléter les propos de Christian Deleuze, le prix maximum accepté par les autorités pour le financement et la prise en charge du médicament orphelin existe finalement déjà aujourd'hui via le dispositif de plafonnement de chiffre d'affaires et les enveloppes contraintes dans lesquelles fonctionnent les organismes payeurs. $\diamond$

\section{LIENS D'INTÉRÊT}

C. Deleuze déclare avoir des liens durables avec l'entreprise SanofiGenzyme.

P. Sarda, L. Druetta, C. Desnuelle, A.S. Lapointe, A. Bernasconi, P.H. Gandon, S. Aymé, N. Bernard, S. Jamot déclarent n'avoir aucun lien d'intérêt concernant les données publiées dans cet article. 